\title{
FIBRE BUNDLE MAPS AND COMPLETE SPRAYS IN FINSLERIAN SETTING
}

\author{
Mircea Crasmareanu
}

\begin{abstract}
A theorem of Robert Blumenthal is used here in order to obtain a sufficient condition for a function between two Finsler manifolds to be a fibre bundle map. Our study is connected with two possible constructions: 1) a Finslerian generalization of usually Kaluza-Klein theories which use Riemannian metrics, the well-known particular case of Finsler metrics, 2) a Finslerian version of reduction process from geometric mechanics. Due to a condition in the Blumenthal's result the completeness of Euler-Lagrange vector fields of Finslerian type is discussed in detail and two situations yielding completeness are given: one concerning the energy and a second related to Finslerian fundamental function. The connection of our last framework, namely a regular Lagrangian having the energy as a proper (in topological sense) function, with the celebrated Poincaré Recurrence Theorem is pointed out.
\end{abstract}

\section{Introduction}

Only suggested by B. Niemann in his "Habilitationvortrag" (1854) and rediscovered by P. Finsler in 1918, the Finsler manifolds became recently a very much alive research domain in differential geometry: [2], [4], [6], [7], [27]. Very interesting and useful for applications in physics and biology, there are some generalizations of Finsler spaces, namely Lagrange and generalized Lagrange spaces, and, more recently, higher-order Finsler and higher-order Lagrange spaces, [28], [29].

In this paper we are concerning with the following:

Question. Decide when a mapping between two Finslerian spaces $\pi:(\tilde{M}, \tilde{L}) \rightarrow$ $(M, L)$ defines a fibre bundle, i.e., for every $p \in M$ there exists a neighborhood $p \in U$ such that $\pi^{-1}(U)$ is diffeomorphic to $U \times \pi^{-1}(p)$. In this case $\tilde{M}, \pi, M$ will be called Finslerian fibre bundle.

Received August 23, 2007.

2000 Mathematics Subject Classification. 55R10, 53C60, 83E15.

Key words and phrases. fibre bundle, fibration, regular Lagrangian, energy, EulerLagrange equations, semispray, spray, Finsler fundamental function, complete vector field, proper function.

Partially supported by CNCSIS Grant No. 583 and CEex Grant 05-D11-84. 
Such a map $\pi$ was called by us a fibre bundle map in the title of this paper, while some authors use the word fibration, e.g. [31, p. 136]. A recent survey in this old problem of determining when a submersion is a fibration or a locally trivial fibre bundle is [26].

The motivation of such a result comes from physics oriented theories. Firstly, the notion of Finsler fundamental function has a variational, more precisely Lagrangian, origin, presented in the next section. Secondly, the treatment by means of fibre bundles appears to be fruitful in order to obtain some remarkable geometrical models for gauge theories. An example only: in Kaluza-Klein's attempts [20] (or its English translations [21], [22]) and [23] (or [24]), for a unified theory, the space-time is the base space of a fibre bundle, more exactly a principal fibre bundle, see also [19]. A main ingredient of the Kaluza-Klein theory is a Riemannian metric and a generalization of Riemannian metrics is provided by Finsler metrics [27]. Therefore it seems that a Finslerian KaluzaKlein theory can be constructed and this paper is intended as a first step in this direction. The first one who pointed out the possibility of a Finslerian Kaluza-Klein theory was R. G. Beil in [8] and [9] and a different approach to this question appears in [34]. For other several applications of Finsler metrics in physics, the cited books offer a good picture, while for a treatment of Finsler and spray geometry in terms of fibre bundles, an excellent survey is [32].

Thirdly, the geometry of fibre bundles serves as main framework in the study of two major problems: i) the integrability of several remarkable dynamical systems; see, for example, [14], ii) the geometrization of control, as it appears, for example, in [10]. Extensions of all these theories, based only on Riemannian metrics, to a Finslerian setting will be an important achievement. Fourthly, a well-known process in geometrical mechanics involving a submersion is the Marsden-Weinstein-Ratiu reduction [25], and then a Finslerian version of this important tool will be useful.

The contents of the paper is as follows. The first section gives a detailed description of the Finsler geometry and a special stress is devoted to obtain global characterizations for the objects of our theory, namely sprays, in order to use the Blumenthal's theorem which is a global result. The second section treats our setting, i.e., Finslerian fibre bundles and a main result is contained in Theorem 2.2. Also, local computations are performed since in physical applications the local coordinates are available. A remark is in order here: although our Theorem 2.2 is a simple consequence of Blumenthal's result we think that the enounce of Theorem 2.2 is more important than an elaborated proof. Using an argument cited above, our Theorem 2.2 can be called Finslerian reduction.

While the Blumenthal characterization is straightforward particularized for Riemannian metrics, leading to the Hermann's fibration theorem from the Riemannian geometry, our result is not, since we need a suitable generalization of the Riemannian submersion concept to the Finsler geometry. To the best of our knowledge, although this question was raised more than ten years ago in [2], a "good" answer does not appears in the literature, except [1]. 
The last section is dedicated to a study of completeness of Euler-Lagrange vector fields, particularly Finslerian sprays, a condition imposed by the Blumenthal's result. Two sufficient conditions for the completeness of a Finslerian spray are presented: firstly in terms of its associate energy and secondly using the Finslerian fundamental function. It is important to note that this second condition involves a proper Finslerian function $F$. It is a remarkable fact that our framework, namely a regular Lagrangian with proper energy, connects with the famous Poincaré Recurrence Theorem, [12, p. 87]. A discussion of the Randers metrics ends the paper.

\section{Finsler geometry revisited}

Let $M$ be a smooth, finite-dimensional manifold with $T M$ and $T^{*} M$ the tangent and cotangent bundle respectively. If $L: T M \rightarrow \mathbb{R}$ is a smooth function, usually called Lagrangian, let $F L: T M \rightarrow T^{*} M$ be the fiber derivative of $L[25$, p. 26]:

$$
F L(v) \cdot w=\left.\frac{d}{d \varepsilon}\right|_{\varepsilon=0} L(v+\varepsilon w)
$$

for $v, w \in T_{p} M, p \in M$. If $\Omega$ denotes the canonical symplectic structure of $T^{*} M$ let $\Omega_{L}=(F L)^{*} \Omega$ be the pullback on $T M$. Let us denote by $C^{\infty}(M)$ the ring of smooth real functions on $M$ and $\mathcal{X}(M)$ the $C^{\infty}(M)$-module of vector fields on $M$.

Definition 1.1 ([25]). (i) The Lagrangian $L$ is called regular if $\Omega_{L}$ is a symplectic structure on $T M$.

(ii) The energy of $L$ is $\mathcal{E}(L): T M \rightarrow \mathbb{R}$ given by:

$$
\mathcal{E}(L)(v)=F L(v) \cdot v-L .
$$

Sometimes the energy appears under the name of Hamiltonian but in our framework being a function on the tangent bundle not on the cotangent bundle we prefer this name. If $L$ is a regular Lagrangian by using the nondegeneracy of the symplectic form $\Omega_{L}$ of $T M$ it result that there exists a unique vector field $S_{L} \in \mathcal{X}(T M)$ such that:

$$
i_{S_{L}} \Omega_{L}=-d \mathcal{E}(L)
$$

where $i_{Z}$ denotes the interior product with respect to the vector field $Z$. $S_{L}$ is called the Euler-Lagrange vector field of $L$ since (1.3) is the global expression of the well-known Euler-Lagrange equations of $L$.

Definition $1.2([27])$. (i) A vector field $S \in \mathcal{X}(T M)$ is called a semispray or a second order differential equation if:

$$
T \tau \circ S=1_{T M},
$$

where $T \tau$ is the differential of the tangent bundle projection $\tau: T M \rightarrow M$ and $1_{T M}$ is the identity of $T M$. 
(ii) A semispray $S \in \mathcal{X}(T M)$ is called a spray if it is positive-homogeneous of order 2 with respect to velocity:

$$
S(a v)=a^{2} \mu_{a, *}(S(v)),
$$

where $a \in \mathbb{R}_{+}, \mu_{t}: T M \rightarrow T M, t \in \mathbb{R}$ is the fibre multiplication (i.e., homotety) by $t$, and $v \in T M$.

Remarks 1.3. (i) The fibre multiplication is the flow of the Euler (or Liouville) vector field $\Gamma=y^{i} \frac{\partial}{\partial y^{i}} \in \mathcal{X}(T M)$ and so, a well-known definition of semisprays in terms of $\Gamma$ and $J=$ the almost tangent structure of $T M$, it follows: $J(S)=\Gamma$.

(ii) In $[13]$ the following characterization of sprays is given: $\left[v_{S}, \Gamma\right]_{F N}=0$ where $v_{S}$ is the vertical projector associated to the semispray $S, v_{S}=\frac{1}{2}\left(1_{\mathcal{X}}(T M)\right.$ $\left.-[J, S]_{F N}\right)$, and $[,]_{F N}$ is the Frölicher-Nijenhuis bracket between a vector 1form, i.e., a tensor field of (1,1)-type, and a vector field.

The first important result is:

Proposition 1.4 ([25], [27]). If $L$ is a regular Lagrangian, then the associated Euler-Lagrange vector field $S_{L}$ is a semispray.

In order to obtain exactly a spray we need:

Definition 1.5 ([27]). A regular Lagrangian $L$ is called Finslerian if:

(i) the following 2-homogeneous condition holds:

$$
L \circ \mu_{a}=a^{2} L
$$

(ii) $L$ is smooth on $T_{0} M$ and continuous on $T M \backslash T_{0} M$, where $T_{0} M$ is the subset of nonvanishing tangent vectors.

It results from $(1.6)$ that the energy $\mathcal{E}(L)$ is 2-homogeneous and applying (1.3) one obtain the main result of this section:

Proposition 1.6 ([27]). If $L$ is Finslerian, then $S_{L}$ is a spray.

Example 1.7. If $g=\left(g_{i j}(x)\right)$ is a Riemannian metric on $M$, then the kinetic energy of $g$ :

$$
L=K(g)=\frac{1}{2} g_{i j} y^{i} y^{j}
$$

is a Finslerian Lagrangian function and $S_{L}$ is the usual geodesic spray which has as projections of integral curves exactly the geodesics of $g$.

For non-Riemannian examples of Finsler functions, namely Randers, Kropina, Matsumoto and others, the reader is invited to browse the bibliography.

\section{Finslerian fibre bundles}

In order to give an answer to the question of Introduction we will use the following fibration theorem due to Robert A. Blumenthal in [11]: 
Theorem 2.1. Let $(\tilde{M}, \tilde{S})$ and $(M, S)$ be two connected manifolds with sprays and let $\pi: \tilde{M} \rightarrow M$ be a submersion. Let $E \subset T \tilde{M}$ be the kernel of $T \pi$ and suppose that there exists $Q \subset T \tilde{M}$ a complementary subbundle of $T \tilde{M}$ (i.e., $T \tilde{M}=E \oplus Q)$ such that:

(i) $Q$ is a union of integral curves of $\tilde{S}$ (in the words of cited paper $Q$ is totally geodesic),

(ii) $\left.\tilde{S}\right|_{Q}$ is T $\pi$-related to $S$.

If $\left.\tilde{S}\right|_{Q}$ is complete, then $\pi$ is onto, $\pi$ is a locally trivial fibre bundle and $S$ is complete.

Remark. Usually, $E$ from above is called the vertical bundle of $\pi$ and is denoted $V(\pi)$ while $Q$ is called a nonlinear connection in [27]. If $M$ and $\widetilde{M}$ are paracompact manifolds then a nonlinear connection exists.

Therefore we are able to give one of the main results of this paper:

Theorem 2.2 (Finslerian Reduction). Let $(\tilde{M}, \tilde{L})$ and $(M, L)$ be two connected Finsler manifolds and let $\pi: \tilde{M} \rightarrow M$ be a submersion. Suppose that there exists $Q \subset T \tilde{M}$ a nonlinear connection on $\pi$ with (i) and (ii) from the previous theorem. If $\left.S_{\tilde{L}}\right|_{Q}$ is complete then $\pi$ is onto, $\pi$ is a locally trivial fibre bundle and $S_{L}$ is complete.

In the case of Riemannian spaces the Blumenthal theorem reduces to the well-known Robert Hermann result about Riemannian fibrations:

Theorem 2.3 ([18], [31, p. 136]). Let $(\tilde{M}, \widetilde{g})$ and $(M, g)$ be two connected Riemannian manifolds and let $\pi: \tilde{M} \rightarrow M$ be a Riemannian submersion. If $(\tilde{M}, \widetilde{g})$ is complete then $\pi$ is a fibre bundle and $(M, g)$ is complete.

The Hermann's result leads to the following:

Question. Which is the natural generalization to Finsler geometry of the notion of Riemannian submersion?

This question appears in the list of open problems of [2] and a partial answer is included in [1].

In the following let us express the Finslerian Reduction in local coordinates. Let $M$ be an $m$-dimensional manifold with $x=\left(x^{i}\right)_{1 \leq i \leq m}$ a local chart and let $(x, y)=\left(x^{i}, y^{i}\right)$ be the adapted chart on $T M$. A semispray $S$ has the expression [27]:

$$
S=y^{i} \frac{\partial}{\partial x^{i}}-S^{i}(x, y) \frac{\partial}{\partial y^{i}}
$$

and $S$ is spray if and only if $S^{i}(x, \lambda y)=\lambda^{2} S^{i}(x, y)$ for every $i$.

Let $\pi: \tilde{M} \rightarrow M$ be a submersion between an $(m+n)$-dimensional manifold and an $m$-dimensional manifold. Then $\pi:\left(x^{i}, \tilde{x}^{a}\right) \rightarrow\left(x^{i}\right)$ and $T \pi$ : $\left(x^{i}, \tilde{x}^{a}, y^{i}, \tilde{y}^{a}\right) \rightarrow\left(x^{i}, y^{i}\right)$ where $1 \leq i \leq m$ and $1 \leq a \leq n$. If $S$ is a semispray 
on $M$ given by (2.1) and $\tilde{S}$ is a semispray on $\tilde{M}$, then the condition (ii) from Theorem 2.1 means:

$$
\tilde{S}=y^{i} \frac{\partial}{\partial x^{i}}+\tilde{y}^{a} \frac{\partial}{\partial \tilde{x}^{a}}-S^{i} \frac{\partial}{\partial y^{i}}-\tilde{S}^{a} \frac{\partial}{\partial \tilde{y}^{a}} .
$$

The kernel of $T \pi$ is $E=\operatorname{span}\left(\frac{\partial}{\partial \tilde{x}^{a}}\right)$ and let $\left(\frac{\delta}{\delta x^{i}}\right)$ be a basis on the nonlinear connection $Q$ which satisfies the Theorem 2.1. From $T \pi\left(\frac{\delta}{\delta x^{i}}\right)=\frac{\partial}{\partial x^{i}}$ it results:

$$
\frac{\delta}{\delta x^{i}}=\frac{\partial}{\partial x^{i}}-B_{i}^{a} \frac{\partial}{\partial \tilde{x}^{a}}
$$

that is $Q=\left(x^{i}, \tilde{x}^{a}, y^{i},-B_{i}^{a} y^{i}\right) \subset T M=\left(x^{i}, \tilde{x}^{a}, y^{i}, \tilde{y}^{a}\right)$. The condition (i) from the Theorem 2.1 implies the following form of the Theorem 2.2:

Theorem 2.4. Let $(\tilde{M}, \tilde{L})$ and $(M, L)$ be connected Finsler manifolds and $\pi: \tilde{M} \rightarrow M$ be a submersion. Suppose that there exists a nonlinear connection $Q$ on $\pi$ such that in each pair of adapted charts $\left(x^{i}\right)$ on $M$ and $\left(x^{i}, \tilde{x}^{a}\right)$ on $\widetilde{M}$ we have:

(i) $Q$ is spanned by $\frac{\delta}{\delta x^{i}}=\frac{\partial}{\partial x^{i}}-B_{i}^{a} \frac{\partial}{\partial \tilde{x}^{a}}$,

(ii) the canonical sprays are given by (2.1) and (2.2) with:

$$
\tilde{S}^{a}(x(t), \tilde{x}(t))=\frac{d}{d t}\left[B_{i}^{a}\left(x(t), \tilde{x}(t), \frac{d x}{d t}(t), \frac{d \tilde{x}}{d t}(t)\right) \frac{d x^{i}}{d t}\right],
$$

where $x(t)$ is a curve on $M$ and $(x(t), \tilde{x}(t))$ is a curve on $\tilde{M}$.

If $\left.S_{\tilde{L}}\right|_{Q}$ is complete, then $\pi$ is onto, $\pi$ is locally trivial fibre bundle and $S_{L}$ is complete.

Let us remark that from (2.4) the 2-homogeneity of the spray $\widetilde{S}$ is provided by the 2-homogeneity of the coefficients $\left(B_{i}^{a}\right)$ of the nonlinear connection $Q$ :

$$
B_{i}^{a}(x, \lambda \widetilde{x}, y, \lambda \widetilde{y})=\lambda^{2} B_{i}^{a}(x, \widetilde{x}, y, \widetilde{y}) .
$$

\section{Completeness of Euler-Lagrange vector fields}

Since a condition in the Theorem 2.2 is regarding to complete sprays this last section is devoted to a study of completeness of Euler-Lagrange vector fields.

Recall that $X \in \mathcal{X}(M)$ is complete if for every $x_{0} \in M$ the maximal interval of existence $\left(t_{-}, t_{+}\right)$for the solution of the flow equation of $X$ with initial condition $x(0)=x_{0}$ is given by $t_{ \pm}= \pm \infty$. A sufficient condition which assures this property is provided by [16] (see also [15] and [35]):

Theorem 3.1. Let $X \in \mathcal{X}(M)$. If there exists $E, f \in C^{\infty}(M)$ with $f$ proper, that is $f^{-1}$ (compact) $=$ compact, and $\alpha, \beta \in \mathbb{R}$ such that for each $x \in M$ we have:

$$
\begin{gathered}
|X(E)(x)| \leq \alpha|E(x)| \\
|f(x)| \leq \beta|E(x)|,
\end{gathered}
$$

then $X$ is complete. 
This has the following important consequence:

Corollary 3.2. Let $S_{L}$ be a Euler-Lagrange vector field. If the energy $\mathcal{E}(L)$ associated to $L$ is proper, then $S_{L}$ is complete.

Proof. Let us take in the previous theorem $E=f=\mathcal{E}(L)$. Since $S_{L}(\mathcal{E}(L))=0$ it follows that all conditions are satisfied with $\alpha=0$ and $\beta=1$.

In this proof we have used the conservation of energy $\mathcal{E}(L)$ along the flow of $S_{L}$, a result which holds for every Euler-Lagrange vector field corresponding to an autonomous, i.e., independent of time, Lagrangian [27]:

Proposition 3.3. If $L=L(x, y)$ is a time-independent Lagrangian, then its energy $\mathcal{E}(L)$ is a first integral of the Euler-Lagrange vector field $S_{L}$.

Example 3.4. If $L$ is a natural Lagrangian, i.e., the difference:

$$
L=K(g)-V,
$$

with $V=V(x)$ a potential, namely a smooth function on $M$, then, according to [15], the Euler-Lagrange vector field $S_{L}$ is complete if the Riemannian metric $g$ is complete and the potential $V$ is bounded below.

Turning to a Finslerian Lagrangian $L$ let us remark that the 2-homogeneity of $\mathcal{E}(L)$ combined with Euler characterization of homogeneous functions implies:

$$
\mathcal{E}(L)=L
$$

which together with the Corollary 3.2 yields:

Corollary 3.5. Let $S_{L}$ be a Finslerian spray. If the Finslerian Lagrangian $L$ is proper, then $S_{L}$ is complete.

Let us recall that the starting point of a Finsler geometry is not the above used Finslerian Lagrangian $L$ but a function $F: T M \rightarrow \mathbb{R}$ such that $L=F^{2}$ is a Finslerian Lagrangian. $F$ is called the Finslerian fundamental function.

If $F$ is proper it results that $F^{2}$ is a proper function too and hence:

Corollary 3.6. Let $S_{L}$ be a Finslerian spray associated to the Finslerian fundamental function $F$ through $L=F^{2}$. If $F$ is a proper function, then $S_{L}$ is complete.

Remark 3.7. i) In fact, the Corollary 3.2, which is the base of the previous result, is the first part of remarkable Poincaré Recurrence Theorem, as it appears in $[12$, p. 87]. A concrete example of a regular Lagrangian with proper energy appears in the same book at page 91 and describes the sliding particle.

ii) In the Finslerian setting a result of Hopf-Rinow type holds in order to characterize topologically the geodesic completeness, [7, p. 168] and Part I of Chapter III from [30]. $[33]$.

iii) An important study of completeness in the Finsler geometry appears in 
Let us point out that there exist two other classes of spray-generating Lagrangians $L$. First is of the form $([5]) L=\varphi\left(F^{2}\right)$ with $F$ a Finslerian fundamental function, but $L$ yields the same Finslerian spray $S_{F}$ as $F$ after the Corollary 2.2 of the cited paper and so we don't have a new situation. The second appears in [3] and contains Lagrangians positively homogeneous of order $m \geq 2$. For this type of Lagrangians it results $\mathcal{E}(L)=(m-1) L$ and we apply the Corollary 3.5.

In order to end with a concrete example let us discuss the case of Randers spaces: a Finslerian fundamental function $F$ is called Randers if it has the form $([7$, p. 17]):

$$
F=\sqrt{a_{i j}(x) y^{i} y^{j}}+b_{i}(x) y^{i},
$$

where $a=\left(a_{i j}\right)$ is a Riemannian metric on $M$ and $b=\left(b_{i}\right)$ is a 1 -form on $M$ with norm with respect to $a$ less than 1 . In [17] it is proved that if $b$ is a closed form then the geodesics of the Finsler-Randers space are exactly the geodesics of Riemannian space $(M, a)$ as point sets. Unfortunately, no information about their parametrization is available to us and so, even if we provide a complete Riemannian metric $a$ it is possible to obtain a non-complete Randers metric!

\section{References}

[1] J. C. Álvarez Paiva and C. E. Durán, Isometric submersions of Finsler manifolds, Proc. Amer. Math. Soc. 129 (2001), no. 8, 2409-2417.

[2] M. Anastasiei, Certain generalizations of Finsler metrics, Finsler geometry (Seattle, WA, 1995), 161-169, Contemp. Math., 196, Amer. Math. Soc., Providence, RI, 1996.

[3] M. Anastasiei and P. L. Antonelli, The differential geometry of Lagrangians which generate sprays, Lagrange and Finsler geometry, 15-34, Fund. Theories Phys., 76, Kluwer Acad. Publ., Dordrecht, 1996.

[4] P. L. Antonelli, R. S. Ingarden, and M. Matsumoto, The Theory of Sprays and Finsler Spaces with Applications in Physics and Biology, Fundamental Theories of Physics, 58. Kluwer Academic Publishers Group, Dordrecht, 1993.

[5] P. L. Antonelli and D. Hrimiuc, A new class of spray-generating Lagrangians, Lagrange and Finsler geometry, 81-92, Fund. Theories Phys., 76, Kluwer Acad. Publ., Dordrecht, 1996.

[6] D. Bao, S.-S. Chern, and Z. Shen, Preface for "Finsler geometry over the reals", Finsler geometry (Seattle, WA, 1995), 3-13, Contemp. Math., 196, Amer. Math. Soc., Providence, RI, 1996.

[7] _ An Introduction to Riemann-Finsler Geometry, Graduate Texts in Mathematics, 200, Springer-Verlag, New York, 2000.

[8] R. G. Beil, Finsler and Kaluza-Klein gauge theories, Internat. J. Theoret. Phys. 32 (1993), no. 6, 1021-1031.

[9]_, Comparison of unified field theories, Tensor (N.S.) 56 (1995), no. 2, 175-183.

[10] A. M. Bloch, Nonholonomic Mechanics and Control, With the collaboration of J. Baillieul, P. Crouch and J. Marsden. With scientific input from P. S. Krishnaprasad, R. M. Murray and D. Zenkov. Interdisciplinary Applied Mathematics, 24. Systems and Control. Springer-Verlag, New York, 2003.

[11] R. A. Blumenthal, Sprays, fibre spaces and product decompositions, Differential geometry (Santiago de Compostela, 1984), 156-161, Res. Notes in Math., 131, Pitman, Boston, MA, 1985. 
[12] R. L. Bryant, An introduction to Lie groups and symplectic geometry, Geometry and quantum field theory (Park City, UT, 1991), 5-181, IAS/Park City Math. Ser., 1, Amer. Math. Soc., Providence, RI, 1995.

[13] M. Craşmăreanu, Nonlinear connections and semisprays on tangent manifolds, Novi Sad J. Math. 33 (2003), no. 2, 11-22.

[14] R. H. Cushman and L. M. Bates, Global aspects of classical integrable systems, Birkhäuser Verlag, Basel, 1997.

[15] D. G. Ebin, Completeness of Hamiltonian vector fields, Proc. Amer. Math. Soc. 26 (1970), 632-634.

[16] W. B. Gordon, On the completeness of Hamiltonian vector fields, Proc. Amer. Math. Soc. 26 (1970), 329-331.

[17] M. Hashiguchi and Y. Ichijyō, Randers spaces with rectilinear geodesics, Rep. Fac. Sci. Kagoshima Univ. No. 13 (1980), 33-40.

[18] R. Hermann, A sufficient condition that a mapping of Riemannian manifolds be a fibre bundle, Proc. Amer. Math. Soc. 11 (1960), 236-242.

[19] _ Yang-Mills, Kaluza-Klein, and the Einstein Program, With contributions by Frank Estabrook and Hugo Wahlquist. Interdisciplinary Mathematics, XIX. Math. Sci. Press, Brookline, Mass., 1978.

[20] T. Kaluza, Zum Unitätsproblem der Physik, Sitzber. Preuss. Akad. Wiss. Kl. 2 (1921), 966-970.

[21] Th. Kaluza, On the problem of unity in physics, Unified field theories of more than 4 dimensions (Erice, 1982), 427-433, World Sci. Publishing, Singapore, 1983.

[22] _ On the unification problem in physics, Translated from the German by $\mathrm{T}$. Muta. An introduction to Kaluza-Klein theories (Deep River, Ont., 1983), 1-9, World Sci. Publishing, Singapore, 1984.

[23] O. Klein, Quantentheorie und fünf-dimensionale Relativitätstheorie, Z. Phys. 37 (1929), 89-901.

[24] _ The Oskar Klein memorial lectures. Vol. 1, Lectures by C. N. Yang and S. Weinberg with translated reprints by O. Klein. Edited by Gosta Ekspong. World Scientific Publishing Co., Inc., River Edge, NJ, 1991.

[25] J. E. Marsden, R. Montgomery, and T. Ratiu, Reduction, symmetry, and phases in mechanics, Mem. Amer. Math. Soc. 88 (1990), no. 436, iv+110 pp.

[26] G. Meigniez, Submersions, fibrations and bundles, Trans. Amer. Math. Soc. 354 (2002), no. 9, 3771-3787.

[27] R. Miron and M. Anastasiei, The Geometry of Lagrange Spaces: Theory and Applications, Fundamental Theories of Physics, 59. Kluwer Academic Publishers Group, Dordrecht, 1994

[28] R. Miron, The Geometry of Higher-Order Lagrange Spaces, Applications to mechanics and physics. Fundamental Theories of Physics, 82. Kluwer Academic Publishers Group, Dordrecht, 1997.

[29] , The Geometry of Higher-Order Finsler Spaces, With a foreword by Ruggero Maria Santilli. Hadronic Press Monographs in Mathematics. Hadronic Press, Inc., Palm Harbor, FL, 1998.

[30] F. Moalla, Sur quelques théorèmes globaux en géométrie finslérienne, Ann. Mat. Pura Appl. (4) 73 (1966), 319-365.

[31] P. Petersen, Riemannian Geometry, Graduate Texts in Mathematics, 171. SpringerVerlag, New York, 1998.

[32] J. Szilasi, A Setting for Spray and Finsler Geometry, Handbook of Finsler geometry. Vol. 1, 2, 1183-1426, Kluwer Acad. Publ., Dordrecht, 2003.

[33] C. Udrişte, Completeness of Finsler manifolds, Publ. Math. Debrecen 42 (1993), no. $1-2,45-50$. 
[34] J. G. Vargas and D. G. Torr, Of Finsler fiber bundles and the evolution of the calculus, Proceedings of the 5th Conference of Balkan Society of Geometers, 183-191, BSG Proc., 13, Geom. Balkan Press, Bucharest, 2006.

[35] A. Weinstein and J. Marsden, A comparision theorem for Hamiltonian vector fields, Proc. Amer. Math. Soc. 26 (1970), 629-631.

Faculty of Mathematics

UNIVERSITY "Al. I. CUZA"

IAŞI, 700506, ROMANIA

E-mail address: mcrasm@uaic.ro 